\title{
On the commutativity degree of metacyclic groups of 5-power order using conjugation action
}

\author{
Siti Norziahidayu Amzee Zamri a, ${ }^{*}$, Nor Haniza Sarmin a, Sanhan Muhammad Salih Khasraw ${ }^{b, c}$, \\ Mustafa Anis El-Sanfaz ${ }^{d}$ and Hamisan Rahmat ${ }^{a}$ \\ a Department of Mathematical Sciences, Faculty of Science, Universiti Teknologi Malaysia, 81310 UTM Johor Bahru, Johor, Malaysia \\ b Salahaddin University-Erbil, College of Education, Department of Mathematics, Kurdistan Region, Iraq \\ c Ishik University-Erbil, Faculty of Education, Mathematics Education Department, Kurdistan Region, Iraq \\ d Institute of Applied Technology, Fatima College of Health Science, United Arab Emirates \\ * Corresponding author: norzisan@gmail.com
}

\section{Article history}

Received 31 May 2017

Accepted 2 October 2017

\begin{abstract}
An extension of the concept of commutativity degree named the probability that an element of a group fixes a set was introduced in 2013. Suppose $G$ is a metacyclic 5-group and $\Omega$ is the set of all ordered pairs $(x, y)$ in $G \times G$ such that $\operatorname{lcm}(|x|,|y|)=5, \quad x y=y x$ and $x \neq y$. In this paper, the probability that an element of a metacyclic 5 -group fixes the set $\Omega$ is computed by using conjugation action.
\end{abstract}

Keywords: Commutativity degree, metacyclic 5-groups, conjugation action

\section{INTRODUCTION}

The probability that a pair of two randomly chosen elements $(x, y)$ from a group $G$ commute is called commutativity degree. The first concept of this probability has been introduced by Erdos and Turan (Erdos and Turan, 1965) in 1965. They investigated several problems based on this concept on symmetric groups. Up until today, this concept has been generalized by various researchers with different scope and groups. In 1973, Gustafson (Gustafson, 1973) showed that the probability of a random pair of elements can be computed by dividing the number of conjugacy classes with the size of the group. He also showed that $P(G) \leq \frac{5}{8}$.

Another extension of the commutativity degree was done by Puornaki and Sobhani (Puornaki and Sobhani, 2008) where they studied the probability that the commutator of two randomly chosen elements in a group is equal to a given element in the same group. Though, this probability was also generalized by Alghamdi and Russo (Alghamdi and Russo, 2012) in 2012 where the upper and lower bound for finite groups were determined. Another work was done by Gastekz (Gastekz, 2010) on solvable and non-solvable groups where two upper bounds on the commutativity degree of non-solvable groups were considered. In 2016, Barzgar (Barzgar, 2016) studied the probability that two subsets of a group commute where several results including lower and upper bounds were obtained.

In this paper, one of the extensions called the probability that a group element fixes a set, $P_{G}(\Omega)$ is used. This probability has been introduced by Omer et al. (Omer et al., 2013) in 2013. In this research, the probability that an element of a metacyclic 5-group fixes a set $\Omega$ is determined by using the conjugation action. In this case, the set $\Omega$ is defined as the set of all ordered pairs $(x, y)$ in $G \times G$ such that $\operatorname{lcm}(|x|,|y|)=5, \quad x y=y x$ and $x \neq y$. This probability is computed by first finding the elements in the group $G \times G$ that satisfy the condition of the set $\Omega$. Later on, the number of the orbits under the same group action on the set $\Omega$ is determined. We will see that the probability that an element of $G$ fixes the set $\Omega$ will eventually depends on the number of the orbits.

\section{METHODOLOGY}

This section provides some basic definitions and previous study that have been done by several authors on the extension of the commutativity degree and probability.

Firstly, some basic definitions on group theory related to this study are given, starting with the definition of metacyclic group.

Definition 2 (Rose, 1944) A group $G$ is called metacyclic if it has a cyclic normal subgroup $H$ such that the quotient group $G / H$ is also cyclic.

Since our work is based on action of the group, the definition of group action is given as follows:

Definition 3 (Rotman, 2003) Let $G$ be any finite group and $X$ be a set. A group $G$ acts on $X$ if there is a function $G \times X \rightarrow X$, such that

i. $\quad(g h) x=g(h x), \forall g, h \in G, x \in X$. 
ii.

$1_{G} x=x, \forall x \in G$.

Since the computation of the orbit is needed before we can find the probability, the definition of the orbit in a group action (specifically conjugation), denoted as $O(x)$, is given in the following definition.

Definition 4 (Goodman, 2006) Let $G$ acts on a set $S$ and $x \in S$. The orbit of $x$ denoted by $O(x)$ is the subset of $S$ where $O(x)=\{g x: g \in G\} \subseteq S$. If a group $G$ acts on itself by conjugation, the orbit $O(x)$ is $y \in G: y=a x a^{-1}$ for some $a \in G$. follows:

In group theory, the conjugacy class of an element is defined as

Definition 5 (Dummit and Foote, 2004) Let $G$ be a finite group. Let $g_{1}, g_{2}$ be the elements of $G$. The elements $g_{1}, g_{2}$ are said to be conjugate if there is some $h$ in $G$ such that $g_{2}=h g_{1} h^{-1}$. The set of all conjugates of $g_{1}$ is called the conjugacy class of $g_{1}$.

In Definition 4, the orbit is also called the conjugacy class of $x$ in $G$.

Throughout this paper, the presentation of the metacyclic $p$-groups is given as in the following theorem:

Theorem 1 (Basri, 2013) Let $G$ be a non-abelian metacyclic $p$ group. Then $G$ is one of the following:

[1] $G \cong\left\langle a, b \mid a^{p^{\alpha}}=b^{p^{\beta}}=1,[b, a]=a^{p^{\alpha-\beta}}\right\rangle$, where $p$ is an odd prime and $\alpha, \beta, \delta \in \square, \delta \leq \alpha<2 \delta, \delta \leq \beta, \delta \leq \min \{\alpha-1, \beta\}$.

[2] $G \cong\left\langle a, b \mid a^{p^{\alpha}}=1, b^{p^{\beta}}=a^{p^{\alpha-\varepsilon}},[b, a]=a^{p^{\alpha-\beta}}\right\rangle$, where $p$ is an odd prime and $\alpha, \beta, \delta, \varepsilon \in \square, \delta+\varepsilon \leq \alpha<2 \delta, \delta \leq \beta$, $\alpha<\beta+\varepsilon, \delta \leq \min \{\alpha-1, \beta\}$.

These two group presentations are classified as groups of Type 1 and Type 2. However, in this paper, only the presentation of metacyclic $p$ groups of Type 1 will be considered, where the value of $p$ is equal to 5 . Next, the extension of the commutativity degree and probability by several authors will be discussed.

In 1975, Sherman (Sherman, 1975) extended the concept of commutativity degree by introducing the probability that an automorphism of a finite group fixes an arbitrary element with the following definition:

Definition 7 (Sherman, 1975) Let $G$ be a group. Let $X$ be a nonempty set of $G$, where $G$ is a permutation group of $X$. Then the probability that an automorphism of a group fixes a random element of $X$ is defined in the following.

$$
P_{G}(X)=\frac{|\{(g, x) \mid g x=x \forall g \in G, x \in X\}|}{|X||G|} .
$$

Later on, Moghaddam et al. (Moghaddam et al., 2011) extended the definition given by Sherman (Sherman, 1975) and introduced a new probability called as the probability that an automorphism of a finite group fixes a subgroup element. This probability is given in the following.

$$
P_{A_{G}}(H, G)=\frac{\left|\left\{(\alpha, h) \mid h^{\alpha}=h, h \in H, \alpha \in A_{G}\right\}\right|}{|H|\left|A_{G}\right|},
$$

where $h$ is a fixed element of $H$ under an arbitrary chosen authomorphism of $G$.

In 2013, Omer et al. (Omer et al., 2013) extended the probability given by Sherman (Sherman, 1975) by introducing the probability that a group element fixes a set. Later in 2016, E-Sanfaz generalized the probability given by omer et al. and introduced the following definition.

Definition 6 (El-Sanfaz, 2016) Let $G$ be a finite group and let $\Omega=\{(a, b) \in G \times G|| a|=| b \mid=2, a b=b a\}$. Let $G$ act on $\Omega$. Then the probability that an element of a group $G$ fixes the set $\Omega$ is given as

$$
P_{G}(\Omega)=\frac{|\{(g, \omega) \in G \times \Omega: g \omega=\omega\}|}{|\Omega||G|} .
$$

Next, the following theorem is used to count the probability that an element of a group fixes a set under the group action of $G$ on $\Omega$.

Theorem 2 (El-Sanfaz, 2016) Let $G$ be a finite group and let $\Omega=\{(a, b) \in G \times G|| a|=| b \mid=2, a b=b a\}$. Let $G$ act on $\Omega$. Then the probability that an element of a group $G$ fixes the set $\Omega$ is given by

$$
P_{G}(\Omega)=\frac{K(\Omega)}{|\Omega|},
$$

where $K(\Omega)$ is the number of orbits under the group action of $G$ on $\Omega$.

In this paper, the probability that an element of a metacyclic 5 -group fixes a set by conjugation action is computed which is given in Theorem 3 .

\section{RESULTS AND DISCUSSION}

This section discusses the main result of this paper which is the probability that an element of a metacyclic 5-group fixes a set by conjugation. First, two lemmas are given, which will be used to proof our main theorem.

Lemma 1 discussed on the elements of order five in the group $G$, presented in the following.

Lemma 1. Let $G$ be a metacyclic 5-group of Type 1 such that $G \cong\left\langle a, b \mid a^{5^{\alpha}}=b^{5^{\beta}}=1,[b, a]=a^{5^{\alpha-\delta}}\right\rangle . \quad$ Then, there are always 24 elements of order five in $G$ which are $a^{5^{\alpha-1} i}, 1 \leq i \leq 4$, $a^{5^{\beta-1} j}, 1 \leq j \leq 4$, and $a^{5^{\alpha-1} i} b^{5^{\beta-1} j}, 1 \leq i \leq 4,1 \leq j \leq 4$.

Proof. Suppose $a$ and $b$ are elements of $G$. Since $a^{5^{\alpha}}=1$ and $b^{5^{\beta}}=1$, thus $|a|$ divides $5^{\alpha}$ and $|b|$ divides $5^{\beta}$. Next, we look at the elements of order five which depends on the value of parameters $\alpha$ and $\beta$. We found that there are four elements of $a \in G$ of order five which are $a^{\alpha^{\alpha-1} i}$ where $1 \leq i \leq 4$, there are four elements of $b \in G$ of order five which are $b^{5^{\beta-1} j}$ where $1 \leq j \leq 4$. Next, there are another 16 elements of order five in terms of $a b \in G$ which are 
$a^{\alpha^{\alpha-1} i} b^{5^{\beta-1} j}$ where $1 \leq i \leq 4,1 \leq j \leq 4$. Thus, there are always 24 elements of order five in $G$, depending on the value of parameters $\alpha$ and $\beta$. The proof then follows.

Next, Lemma 2 discussed on the size and elements in the set $\Omega$, given in the following.

Lemma 2. Let $G$ be a metacyclic 5-group of Type 1 such that $G \cong\left\langle a, b \mid a^{5^{\alpha}}=b^{5^{\beta}}=1,[b, a]=a^{5^{\alpha-\delta}}\right\rangle$ and the set $\Omega$ is an ordered set set of $G \times G$ in the form of $(x, y)$ such that $\operatorname{lcm}(|x|,|y|)=5$, $x y=y x$, and $x \neq y$. Then $|\Omega|=300$.

Proof. Suppose $G$ is a metacyclic 5-group of Type 1 such that $G \cong\left\langle a, b \mid a^{5^{\alpha}}=b^{5^{\beta}}=1,[b, a]=a^{5^{\alpha-\delta}}\right\rangle$ and the set $\Omega$ is an ordered set of $G \times G$ in the form of $(x, y)$ such that $\operatorname{lcm}(|x|,|y|)=5, \quad x y=y x$ and $x \neq y$. By Lemma 1 , there are 24 elements of order five such that $a^{5^{\alpha-1} i}, 1 \leq i \leq 4, \quad b^{5^{\beta-1} j}, 1 \leq j \leq 4, \quad a^{5^{\alpha-1} i} b^{5^{\beta-1} j}, 1 \leq i \leq 4,1 \leq j \leq 4$. Since $\operatorname{lcm}(|x|,|y|)=5$, we need to consider the pair $(|x|,|y|)$ of order $|x|=1$ and $|y|=5$, and $|x|=5$ and $|y|=5$. Since the identity commutes with all elements of $g \in G$, we first have 24 pairs $(x, y)$ when $|x|=1$ and $|y|=5$. Next, we check whether the pair $(x, y)$ when $|x|=5$ and $|y|=5$ commutes with each other or not, and we found that all pairs commute with each other. This gives us $24 \times 24=576$ pairs. However, since $x \neq y, 288$ pairs that gives the same $(x, y)$ pairs, and the same $(x, x)$ and $(y, y)$ pairs need to be omitted. Thus, we are left with another 276 pairs. In total, we have $276+24=300$ ordered pairs of elements in the set $\Omega$. The proof then follows.

Theorem 3. Let $G$ be a metacyclic 5-group of Type 1 such that $G \cong\left\langle a, b \mid a^{5^{\alpha}}=b^{5^{\beta}}=1,[b, a]=a^{5^{\alpha-\delta}}\right\rangle$ where $\alpha, \beta, \delta \in \square, \delta \leq \alpha<2 \delta$, $\delta \leq \beta, \quad \delta \leq \min \{\alpha-1, \beta\}$ and $\Omega$ the set of ordered pairs $(x, y)$ in $G \times G$ such that $\operatorname{lcm}(|x|,|y|)=5, \quad x y=y x$ and $x \neq y$. If $G$ acts on $\Omega$ by conjugation, then

$$
P_{G}(\Omega)=\left\{\begin{array}{lr}
\frac{17}{75}, & \text { when } \alpha>\beta=\delta, \\
1, & \text { otherwise. }
\end{array}\right.
$$

Proof. Suppose $G$ acts on $\Omega$ by conjugation, then there exists a function $\psi: G \times \Omega \rightarrow \Omega$ such that $\psi_{g}(\omega)=g \omega g^{-1}, \omega \in \Omega, g \in G$. By Lemma 2 , there are 300 ordered pairs of elements $\left(\omega_{1}, \omega_{2}\right)$ in $\Omega$. Thus, there are 300 orbits, $O\left(\omega_{1}, \omega_{2}\right)=g\left(\omega_{1}, \omega_{2}\right) g^{-1}, \forall g \in G$ that needs to be computed. Based on the parameters $\alpha, \beta$ and $\delta$, it has been found that the value for parameters $\alpha, \beta, \delta$ can only be divided into two cases, the first one is when $\alpha>\beta=\delta$, and the other one when $\alpha>\beta>\delta, \alpha=\beta>\delta$, and $\alpha<\beta>\delta$. Therefore, the value of the orbits are also depending on these two cases.

Case 1. When $\alpha>\beta=\delta$. For instance, let $\alpha=3, \beta=2$ and $\delta=2$. Thus, we have $G \cong\left\langle a, b \mid a^{125}=b^{25}=1,[b, a]=a^{5}\right\rangle$, and $|G|=3125$. From the computation of 300 ordered pairs of $\Omega$, it was found that there are 10 orbits of size one, which are $\left(1, a^{100}\right),\left(1, a^{75}\right),\left(1, a^{50}\right)$, $\left(1, a^{25}\right), \quad\left(a^{100}, a^{75}\right), \quad\left(a^{100}, a^{50}\right), \quad\left(a^{100}, a^{25}\right), \quad\left(a^{75}, a^{50}\right),\left(a^{75}, a^{25}\right)$, $\left(a^{50}, a^{25}\right)$. Since the identity element and $a^{100}, a^{75}, a^{50}$ and $a^{25}$ are in the center of $G$, the orbits $O\left(\omega_{1}, \omega_{2}\right)=g\left(\omega_{1}, \omega_{2}\right) g^{-1}=g g^{-1}\left(\omega_{1}, \omega_{2}\right)$ are themselves, which is of size one. Next, we found that there are 58 orbits of size five which starts with $\left(1, b^{5}\right),\left(1, b^{10}\right), \ldots,\left(b^{20}, a^{75} b^{20}\right)$. Thus, we have a total of 68 orbits which is the subset of $\Omega$. By Theorem 2, $P_{G}(\Omega)=\frac{K(\Omega)}{|\Omega|}=\frac{68}{300}=\frac{17}{75}$.

Case 2. When $\alpha>\beta>\delta, \alpha=\beta>\delta$, and $\alpha<\beta>\delta$. For instance, take the value when $\alpha=\beta>\delta$ and let $\alpha=3, \beta=3, \delta=2$. Thus, we have $G \cong\left\langle a, b \mid a^{125}=b^{125}=1,[b, a]=a^{5}\right\rangle$, and $|G|=15625$. It was found that all elements of order one and five, which are the identity element, $\quad a^{5^{\alpha-1} i}, 1 \leq i \leq 4, \quad b^{5^{\beta-1} j}, 1 \leq j \leq 4$, $a^{5^{\alpha-1} i} b^{5^{\beta-1} j}, 1 \leq i \leq 4,1 \leq j \leq 4$, are in the center of $G$. Therefore, the orbits $O\left(\omega_{1}, \omega_{2}\right)=g\left(\omega_{1}, \omega_{2}\right) g^{-1}=g g^{-1}\left(\omega_{1}, \omega_{2}\right)$ are themselves, which are of size one. The pairs are also similar as the ordered pairs of $\Omega$. By Theorem 2, $P_{G}(\Omega)=\frac{|\Omega|}{|\Omega|}=\frac{68}{68}=1$. After the computation, it was found that similar value is applicable to cases when $\alpha>\beta>\delta$ and $\alpha<\beta>\delta$, as well. The proof then follows.

A software called Groups, Algorithms and Programming (GAP) has been used in this research to facilitate our computation in finding the order of elements in the group $G$, since the size of each group are large and it takes so much time to do hand computation. We give an example of GAP code based on the case when $\alpha=3, \beta=2, \delta=2$. The GAP codes are given as follows:

f:=FreeGroup("a","b");;

$\mathrm{a}:=\mathrm{f} .1 ; ; \mathrm{b}:=\mathrm{f} .2 ;$

$r:=\left[a^{\wedge} 125, b^{\wedge} 25, \operatorname{Comm}(b, a) * a^{\wedge}-5\right] ; ;$

$\mathrm{g}:=\mathrm{f} / \mathrm{r}$;

a:=g. $1 ;$; $:=$ g. $2 ;$;

Size $(\mathrm{g})$;

3125

e:=Elements(g);;

for $\mathrm{i}$ in $[1 . . \operatorname{Size}(\mathrm{g})]$ do

els:=e[i];

$\mathrm{p}:=\operatorname{Order}(\mathrm{e}[\mathrm{i}])$;

Print(els, " ", p , " ", "\n");

od;

e3:=Filtered(e, c->Order(c)=3);

$\operatorname{Add}(\mathrm{e} 3$,Identity(g));

e13:=AsSet(e3);

$\mathrm{A}:=[] ;$

for i in [1..Size(e13)-1] do

$\mathrm{a}:=\mathrm{e} 13[\mathrm{i}]$;

for $\mathrm{j}$ in $[\mathrm{i}+1 . . \operatorname{Size}(\mathrm{e} 13)] \mathrm{do}$

$\mathrm{b}:=\mathrm{e} 13[\mathrm{j}]$;

if $\operatorname{Comm}(\mathrm{a}, \mathrm{b})=\operatorname{Identity}(\mathrm{g})$ then

$\operatorname{Add}(\mathrm{A},[\mathrm{a}, \mathrm{b}])$

fi;

od;

od;

$\mathrm{B}:=[] ;$

for $\mathrm{i}$ in $[1 . . \operatorname{Size}(\mathrm{A})] \mathrm{do}$

$\mathrm{C}:=[]$;

if not $\mathrm{A}[\mathrm{i}]$ in $\mathrm{B}$ then

for $g g$ in e do

for $\mathrm{j}$ in $[1 . . \operatorname{Size}(\mathrm{B})] \mathrm{do}$ 


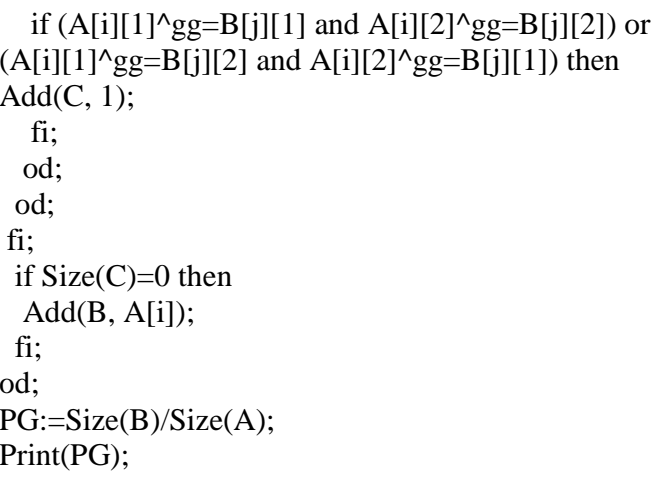

\section{CONCLUSION}

In this paper, the probability that an element of a group fixes a set is found for metacyclic 5-groups of Type 1. The result shows that the probability depends on the size of the orbits and the size of the set $\Omega$. It was also found that there are only two cases of the value of the probability, which are also depending on the value of parameters $\alpha, \beta$ and $\delta$.

\section{ACKNOWLEDGEMENT}

The authors would like to express their gratitude to the Ministry of Higher Education Malaysia (MOHE) and Universiti Teknologi Malaysia for the funding through research grant Vote No. 13H79 and 11J96. The first author is also indebted to MOHE with her MyPhD scholarship.

\section{REFERENCES}

Erdos, P. and Turan, P. 1965. On some problems of a statistical group theory. Probability Theory and Related Fields. 4(2), 175-186.

Gustafson, W. 1973. What is the probability that two group elements commute? The American Mathematical Monthly. 80(9), 1031-1034.

Pournaki, M. R. and Sobhani, R. 2008. Probability that commutator of two group elements is equal to a given element. Journal of Pure and Applied Algebra. $212,727-734$.

Alghamdi, A. M. and Russo, F. G. 2012. A Generalized of the probability that commutator of two group elements is equal to a given element. Bulletin of Iranian Mathematical Society. 38(4), 973-986.

Gastekz, A. and Kuzmanovich, J. 2010. Commutativity Degree of a Finite Group. Ms.C. Wake Forest University, North Carolina.

Barzgar, R., Erfanian, A. and Farrokhi, M. 2016. Probability of mutually commuting two subsets of a finite group. Ars Combinatoria. CXXIV, 165176 .

Omer, S. M. S., Sarmin, N. H., Erfanian, A. and Moradipour, K. 2013. The probability that an element of a group fixes a set and the group act on set by conjugation. International Journal of Applied Mathematics and Statistics. 32(2), 111-117.

El-Sanfaz, M. A. 2016. The probability that an element of a non-abelian group fixes a set and its applications in graph theory, Doctor of Philosophy Thesis, Universiti Teknologi Malaysia.

Rose, J. S. 1978. A course on group theory. Great Britain, Cambridge:

Cambridge University Press.

Rotman, J. J. 2002. Advanced modern algebra. Upper Saddle River, New Jersey:

Prentice Hall.

Goodman, F. 2003. Algebra: abstract and concrete. ( $2^{\text {nd }}$ Edition). Upper Saddle

River, New Jersey: Prentice Hall.

Dummit, D. S. and Foote, R. M. 2004. Abstract algebra. ( $3^{\text {rd }}$ Edition). United

States of America: John Wiley \& Sons, Inc.

Basri, A. M. A. 2013. Capability and homological functors of finite metacyclic p-groups. Doctor of Philosophy Thesis. Universiti Teknologi Malaysia.

Sherman, G. 1975. What is the probability an automorphism fixes a group element? The American Mathematical Monthly. 82(3), 261-264.

Moghaddam, M. R. R. Saeedi, F. and Khamseh, E. 2011. The probability of an automorphism fixing a subgroup element of a finite group. Asian-European Journal of Mathematics. 4(2), 301-308. 